\title{
Clinical and Social Determinants to IMPROVE DOD/VETERAN WELL BEING: THE SERViCE MEMBER VETERAN RisK Profile
}

\author{
Richard T. Hartman ${ }^{1}$ and Mark E. Oxley ${ }^{2}$ \\ ${ }^{1}$ Department of Veterans Affairs, Washington DC, USA \\ ${ }^{2}$ Department of Mathematics and Statistics, Graduate School of Engineering and \\ Management, Air Force Institute of Technology, Wright-Patterson AFB, Ohio, USA
}

\begin{abstract}
This paper introduces the Service member Veteran Risk Profile (SVRP), a mathematical process/solution to quantitatively represent transitioning Service member (TSM) and/or Veteran quality of life risks by integrating clinical and social determinant data into an individual risk profile. The SVRP creates, for the first time, a mechanism for the Department of Defense (DoD) and Department of Veterans Affairs (VA) to holistically represent the challenges of military members transitioning into civilian life that can lead to negative outcomes and proactively identify transitioning Service members and Veterans at risk. More importantly, the SVRP supports clinical and non-clinical modalities to reduce the negative impacts of transition and beyond for TSM and Veterans. Lastly, the SVRP can be displayed through user-friendly visualizations so DoD/VA policymakers and decision-makers can make more informed policy and resource decisions to improve TSM/Veteran overall quality of life.
\end{abstract}

\section{KEYWORDS}

Clinical determinants, social determinants, healthcare, health, suicide, public policy, personalized medicine, informatics, veterans, Big Data, homelessness

\section{INTRODUCTION}

Recent research suggests transition from active duty to civilian life is one period of stress that has critical impact on the future functioning and quality of life of the Veteran [1]. These transition challenges are associated with negative outcomes, such as, homelessness, suicide ideation, and first episode psychosis [2].

To address the potential negative outcomes of transition, Department of Defense (DoD) and Department of Veterans Affairs (VA) clinicians currently rely heavily on clinical determinants to assess transitioning Service member (TSM)/Veteran health and clinical treatments to mitigate negative outcomes, even though there are many risk factors beyond the clinical determinants of health that contribute to the aforementioned negative outcomes [3].

To help stem the increased prevalence of negative outcomes associated with transition to civilian life, such as suicide [5], in the age of informatics and Big Data, DoD and VA needed a way to identify, collate, and address the complexities associated with military to civilian transition and mitigate the negative outcomes from transition to civilian life: a novel approach combining clinical and social determinant data into insight and ultimately action. 
To respond to this need, a mathematical process/solution was developed that not only integrated clinical and social determinants but provided the DoD/VA with a process for clinicians, social benefits advisors, and decision makers that could: 1) identify the factors associated with negative outcomes, such as suicide, homelessness, unemployment, and financial strain into a single quantitative risk, 2) provide clinical and/or social service mitigation strategies to address TSM/Veteran risks and 3) aggregate individual TSM/Veteran data into population formats to inform decision makers on key policy and resource decisions. Furthermore, they needed a dynamic approach that could evolve with advances in sciences and informatics. This new approach is called the Service member Veteran Risk Profile (SVRP).

\section{Derivations}

The SVRP leverages advances in the clinical and social sciences and informatics allowing data scientist to integrate disparate and unstructured data from both clinical and nonclinical data sources and account for the many factors that contribute to negative outcomes TSM/Veterans face associated with transition to civilian life [6]. The SVRP provides clinicians and social service providers the ability to visualize and quantify a transitioning Service member or Veteran risks beyond any one variable. This new methodology, when applied, turns multiple sources of clinical and social determinant data into actionable insight for healthcare/social benefits professionals through a holistic risk profile.

This holistic risk profile first required the development of a mathematical expression (i.e., equation), and processes so the disparate and unstructured data collected from clinical and nonclinical records could be merged into a numerical representation of an individual's overall risk from negative outcomes.

To build the SVRP, our initial mathematical expression was created based on a single notional negative outcome with a set of three notional factors that could contribute to the overall negative outcome. This is expressed as the Service member Veteran Risk Index (SVRI).

$$
\mathrm{SVRI}_{1}=v_{l, 1}+v_{l, 2}+v_{l, 3}
$$

Here $v_{1,1}$ is the value of the individual's poverty status. The value $v_{1,2}$ is the same individual 1 , and the 2 represents the unemployment status. The $v_{1,3}$ is also the same individual 1 , and 3 represents the clinical effect per the evidence in the individual's medical record (clinical history).

To account for more than one negative outcome, we simply combined several negative outcomes or Service member Veteran Risk Indices (SVRIs) to create the SVRP, which is expressed as a column vector [7],

$$
\mathrm{SVRP}=\left[\begin{array}{c}
S V R I_{1} \\
\vdots \\
S V R I_{M}
\end{array}\right] .
$$

\subsection{Classifier Function for the SVRP}

Generating an overall numerical and/or visual representation of risk necessitated the creation of a risk value, that is, a numerical value for each variable $v_{\mathrm{m}, \mathrm{n}}$ within the SVRP. The lower case $m$ is a variable representing an individual's negative outcome, such as homelessness, suicide ideation, etc., and the lower case $n$ represents confounding factors associated with that risk, such as, poverty status, medical condition, etc. That is, if the confounding factor for the negative outcome of the 
individual was determined to be "at risk" or "not at risk" then the corresponding numerical values for $v_{\mathrm{m}, \mathrm{n}}$ would be either 1 or 0 , respectively.

For example, let us begin with a generic risk value and conclude with a single risk when compared to a risk threshold. Suppose there is a test $T$ related to a risk, and this test is performed on the individual, $h$. Suppose the output of the test is a single numerical value, $r$, called the test measurement value. Since test $T$, acting on individual, $h$, yields the value, $r$, then we will write it in function notation as $T(h)=r$.

We seek a classifier $C$ whose input is the test measurement value $r$ and output is a risk value $v$, that is, a real number. The possible values for $r$ could be any real number that is compared to a threshold value where an $r_{\mathrm{i}}$ to the threshold yields a $v=1$ and a threshold yields a $v=0$, that is, $v \in[0,1]$.

The classifier $C$ should be a function such that $C(r)=v$. Notice that composing the test function, $\mathrm{T}$, with the classifier function, $C$, applied to $\mathrm{v} \in\{0,1\}$ individual, h, yields the risk value $v$, that is $C(\mathrm{~T}(\mathrm{~h}))=v$. Many classifiers are often simplified such that the risk values are either 1 or 0 , where the value $v=1$ corresponds to the label "at risk" and the value $v=0$ corresponds to "not at risk".

There are many tests where the classifier $C$ already exists as an established classifier based upon studies with data from a population of individuals, such that a threshold value, $\theta$, exists (that is, a real number called the standard threshold value) and is defined to be

$$
v=C(r)= \begin{cases}0, \text { if } & r<\theta \\ 1, \text { if } & r \geq \theta\end{cases}
$$

For some tests the measurement values presented to the classifier could be defined such that

$$
v=C(r)= \begin{cases}1, \text { if } & r<\theta \\ 0, \text { if } & r \geq \theta\end{cases}
$$

A practical example of interest is Suicide Ideation (SI). For example, when evaluating SI, Equation (1) becomes

$$
\mathrm{SVRI}_{(\mathrm{SI})}=\mathrm{V}_{(\mathrm{SI} \text {, poverty status })}+\mathrm{v}_{(\mathrm{SI} \text {, employment status })}+\mathrm{V}_{(\mathrm{SI}, \text { medical history })}
$$

The threshold for poverty status, according to the US Department of Health and Human Service (DHHS), is $\$ 12,060$ for a single person in family/household [8]. Therefore, the classifier function for the poverty status measurement $r_{1,1}$ with a threshold value of $\$ 12,060$ is

$$
v_{1,1}=C\left(\mathrm{r}_{1,1}\right)=\left\{\begin{array}{l}
0, \text { if } \mathrm{r}_{1,1}<\$ 12,060 \\
1, \text { if } \mathrm{r}_{1,1} \geq \$ 12,060 .
\end{array}\right.
$$

Hence, if the measured value $r_{1,1}$ of the poverty status was greater than $\$ 12,060$ then the variable $v_{l, 1}$ would be recorded as 1 , and if the measured value $\mathbf{r}_{1,1}$ of the poverty status was less than $\$ 12,060$, then the variable $v_{l, l}$ would be recorded as 0 .

Recognizing that not all SVRI variables, v, can be defined by Equations (3) and (4), it became evident that an additional generic classifier function was needed for $v$ when no validated/credible threshold value, $\theta$ existed. This classifier function based on presence/absence can be expressed as: 


$$
v=C(r)=\left\{\begin{array}{l}
1, \text { if } r=\text { presence } \\
0, \text { if } r=\text { absence }
\end{array}\right.
$$

where $r$ may or may not be a numerical value. That is, if $r=$ present then $v=1$ and if $r \neq$ present then a $v=0$, that is, $v \in\{0,1\}$. For example, when evaluating SI and the risk factor $v_{1,2}$ associated with unemployment status, we know that unemployment can be detrimental to mental health and is associated with increased risk of suicide [8]. That is, if

$$
\mathrm{v}_{1,2}=C\left(\mathrm{r}_{1,2}\right)=\left\{\begin{array}{l}
1, \text { if } \mathrm{r}_{1,2}=\text { presence of unemployment } \\
0, \text { if } \mathrm{r}_{1,2}=\text { absence of unemloyment }
\end{array}\right.
$$

then, if the unemployment $r_{1,2}$ is present the variable $v_{1,2}$ would be recorded as 1 , and if the unemployment $r_{1,2}$ is absent then the variable $v_{1,2}$ would be recorded as 0 .

With the classifier functions developed to populate variables $\left(v_{l, l}, v_{l, 2}, v_{l, 3}\right)$ for equation (1) then the SVRI can produce a meaningful value of risk, and recognizing the SVRP as a combination of SVRI's for an individual transitioning Service member or Veteran, then equation (2) can now be expressed as

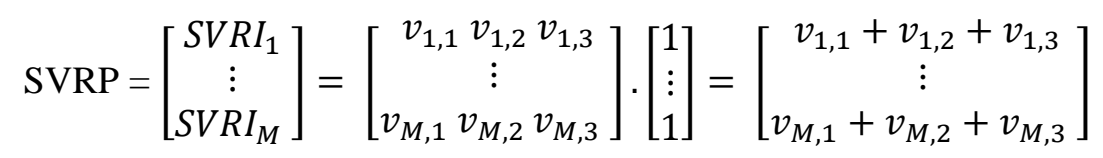

to account for multiple exposures, where $v_{m, 1}$ is now $v_{M, l}$ after the conversion of the raw data $m$ to the classifier result $M$.

\section{EXTENSION OF MULTIPLE DETERMINANTS OF THE SVRP}

With the SVRP equipped to address multiple negative outcomes and only three variables to describe an individual negative outcome like homelessness and/or suicide, the limitations to the SVRI became evident. This required the SVRI to be modified so it could account for multiple confounding factors (see Figure 1), such as, family history, health access, and education, and is represented by the Equation (10) with $N$ confounding factors or determinants [6].

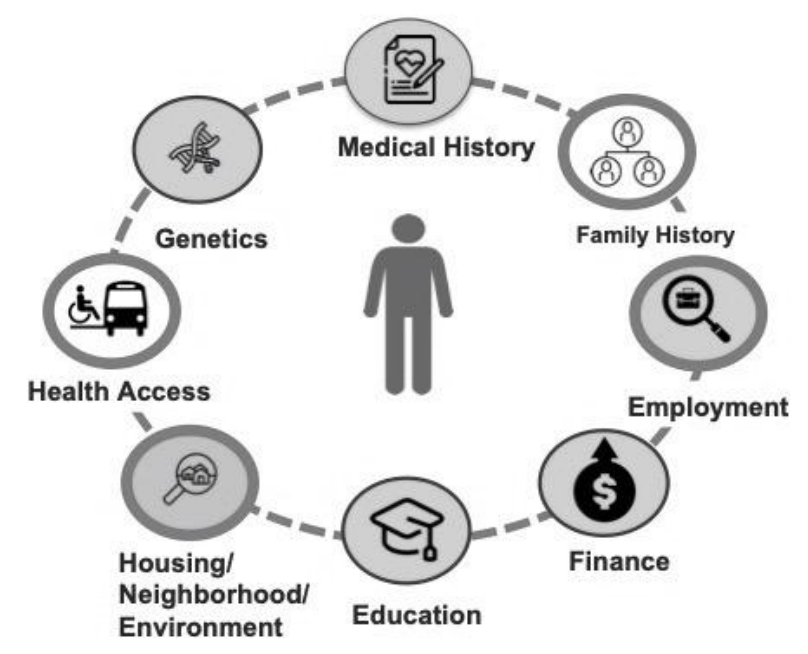

Figure 1. Service Member Veteran Risk Index can have many variables. 


$$
\operatorname{SVRP}=\left[\begin{array}{c}
S V R I_{1} \\
\vdots \\
S V R I_{M}
\end{array}\right]=\left[\begin{array}{ccc}
v_{1,1} & \cdots & v_{1, N} \\
\vdots & \ddots & \vdots \\
v_{M, 1} & \cdots & v_{M, N}
\end{array}\right] \cdot\left[\begin{array}{c}
1 \\
\vdots \\
1
\end{array}\right]=\left[\begin{array}{c}
v_{1,1}+\cdots+v_{1, N} \\
\vdots \\
v_{M, 1}+\cdots+v_{M, N}
\end{array}\right]
$$

\subsection{Visualization and Normalization of the SVRP}

To visualize the SVRP, let us assume we have an SVRP for an individual with four separate negative outcomes. Applying classifier functions to notional raw data for every $r_{m, n}$ we get the following values for each $v_{m, n}$ in the matrix array, where the SVRP is expressed as a column vector in Equation (11),

$$
\text { SVRP }=\left[\begin{array}{l}
\text { SVRI1 } \\
\text { SVRI2 } \\
\text { SVRI3 } \\
\text { SVRI4 }
\end{array}\right]=\left[\begin{array}{lll}
1 & 1 & 1 \\
1 & 0 & 1 \\
0 & 0 & 1 \\
0 & 0 & 1
\end{array}\right] \cdot\left[\begin{array}{l}
1 \\
1 \\
1
\end{array}\right]=\left[\begin{array}{l}
3 \\
2 \\
1 \\
1
\end{array}\right]
$$

And represented visually in Figure 2.

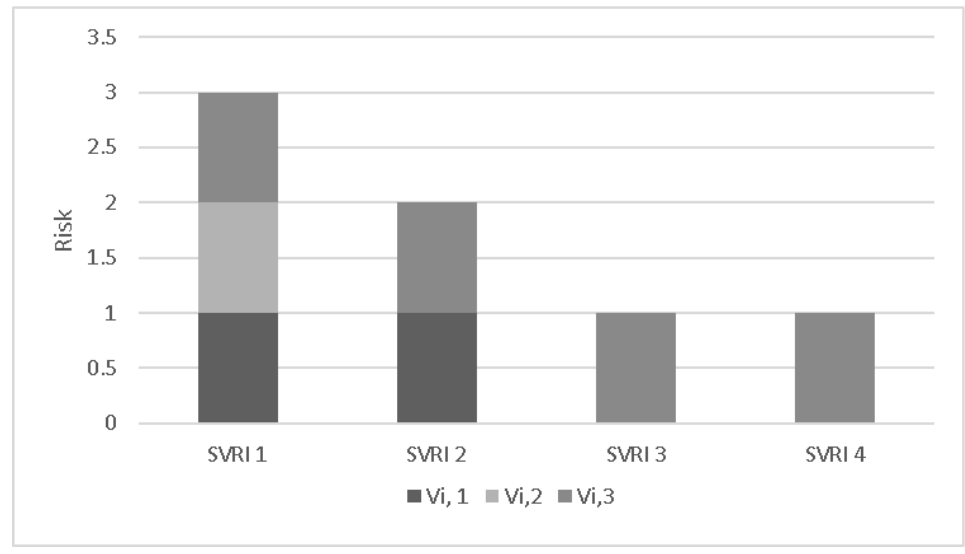

Figure 2. Visualizing SVRP using notional raw data for an individual transitioning Service member/Veteran.

To ensure each SVRP could be represented in a common scale for future comparison it became necessary to normalize the output of (11) such that each SVRI was displayed between 0 and 1 . This resulted in the following equation

$$
\operatorname{SVRP}=\left[\begin{array}{c}
S V R I_{1} \\
\vdots \\
S V R I_{M}
\end{array}\right]=\left[\begin{array}{ccc}
\left(\mathrm{v}_{1,1}\right)\left(\mathrm{NF}_{1,1}\right) & \cdots & \left(\mathrm{v}_{1, \mathrm{~N}}\right)\left(\mathrm{NF}_{1, \mathrm{~N}}\right) \\
\vdots & \ddots & \vdots \\
\left(\mathrm{v}_{\mathrm{M}, 1}\right)\left(\mathrm{NF}_{\mathrm{M}, 1}\right) & \cdots & \left(\mathrm{v}_{\mathrm{M}, \mathrm{N}}\right)\left(\mathrm{NF}_{\mathrm{M}, \mathrm{N}}\right)
\end{array}\right] \cdot\left[\begin{array}{l}
1 \\
1 \\
1
\end{array}\right] .
$$

Simplifying the notation by observing the different factors form their own matrices, we rewrite Equation (12) as

$$
S V R P=\left[\begin{array}{c}
S V R I_{1} \\
\vdots \\
S V R I_{M}
\end{array}\right]=\left(\left[\begin{array}{ccc}
v_{1,1} & \cdots & v_{1, N} \\
\vdots & \ddots & \vdots \\
v_{M, 1} & \cdots & v_{M, N}
\end{array}\right] \odot\left[\begin{array}{ccc}
N F_{1,1} & \cdots & N F_{1, N} \\
\vdots & \ddots & \vdots \\
N F_{M, 1} & \cdots & N F_{M, N}
\end{array}\right]\right) \cdot\left[\begin{array}{c}
1 \\
\vdots \\
1
\end{array}\right]
$$


Where the symbol $\odot$ denotes the Hadamard product of two matrices of the same size [10]. Therefore, the SVRP in its simplest form is

$$
S V R P=[V \odot N F] \cdot[\mathbf{1}]
$$

Where

$$
V=\left[\begin{array}{ccc}
v_{1,1} & \cdots & v_{1, N} \\
\vdots & \ddots & \vdots \\
v_{M, 1} & \cdots & v_{M, N}
\end{array}\right], N F=\left[\begin{array}{ccc}
N F_{1,1} & \cdots & N F_{1, N} \\
\vdots & \ddots & \vdots \\
N F_{M, 1} & \cdots & N F_{M, N}
\end{array}\right] \quad \text { and }[\mathbf{1}]=\left[\begin{array}{c}
1 \\
\vdots \\
1
\end{array}\right]
$$

Using data in the previous example in conjunction with Equation (13), results in the following $M$ $=4$ and $N=3$ :

$$
S V R P=\left[\begin{array}{l}
\text { SVRI1 } \\
\text { SVRI2 } \\
\text { SVRI3 } \\
\text { SVRI4 }
\end{array}\right]=\left(\left[\begin{array}{lll}
1 & 1 & 1 \\
1 & 0 & 1 \\
0 & 1 & 1 \\
0 & 0 & 1
\end{array}\right] \odot\left[\begin{array}{ccc}
\frac{1}{3} & \frac{1}{3} & \frac{1}{3} \\
\frac{1}{3} & 0 & \frac{1}{3} \\
0 & \frac{1}{3} & \frac{1}{3} \\
0 & 0 & \frac{1}{3}
\end{array}\right]\right) \cdot\left[\begin{array}{l}
1 \\
1 \\
1
\end{array}\right]=\left[\begin{array}{ccc}
\frac{1}{3} & \frac{1}{3} & \frac{1}{3} \\
\frac{1}{3} & 0 & \frac{1}{3} \\
0 & \frac{1}{3} & \frac{1}{3} \\
0 & 0 & \frac{1}{3}
\end{array}\right] \cdot\left[\begin{array}{c}
1 \\
1 \\
1
\end{array}\right]=\left[\begin{array}{c}
1 \\
66 \\
66 \\
.33
\end{array}\right]
$$

This result is represented visually in Figure 3 where each SVRI is displayed between 0 and 1.

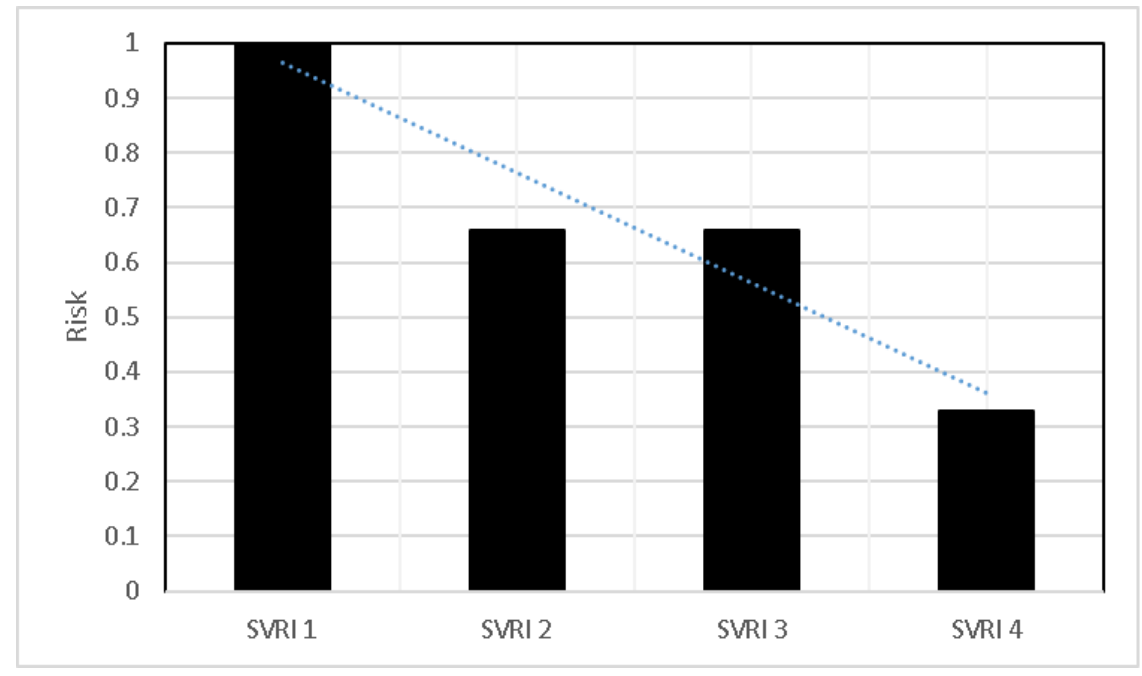

Fig. 3. Visualizing SVRP after normalizing notional raw data for an individual.

\subsection{Censored Data}

Recognizing there may be instances where raw data may be not available (e.g., no financial data, missing or lost medical records, etc.), the issue of censored data became evident, since one could not assume a missing value is 1 or 0 with confidence. This issue can be easily demonstrated by replacing the 0 's in (16) with dashes, where (-) denotes censored data resulting in: 


$$
S V R P=\left[\begin{array}{l}
\text { SVRI1 } \\
\text { SVRI2 } \\
\text { SVRI3 } \\
\text { SVRI4 }
\end{array}\right]=\left(\left[\begin{array}{ccc}
1 & 1 & 1 \\
1 & - & 1 \\
- & - & 1 \\
- & - & 1
\end{array}\right] \odot\left[\begin{array}{ccc}
\frac{1}{3} & \frac{1}{3} & \frac{1}{3} \\
\frac{1}{2} & 0 & \frac{1}{2} \\
0 & \frac{1}{2} & \frac{1}{2} \\
0 & 0 & 1
\end{array}\right]\right) \cdot\left[\begin{array}{l}
1 \\
1 \\
1
\end{array}\right]=\left[\begin{array}{ccc}
\frac{1}{3} & \frac{1}{3} & \frac{1}{3} \\
\frac{1}{2} & 0 & \frac{1}{2} \\
0 & \frac{1}{2} & \frac{1}{2} \\
0 & 0 & 1
\end{array}\right] \cdot\left[\begin{array}{l}
1 \\
1 \\
1
\end{array}\right]=\left[\begin{array}{l}
1 \\
1 \\
1 \\
1
\end{array}\right]
$$

where the end result is represented visually by Figure 4 .

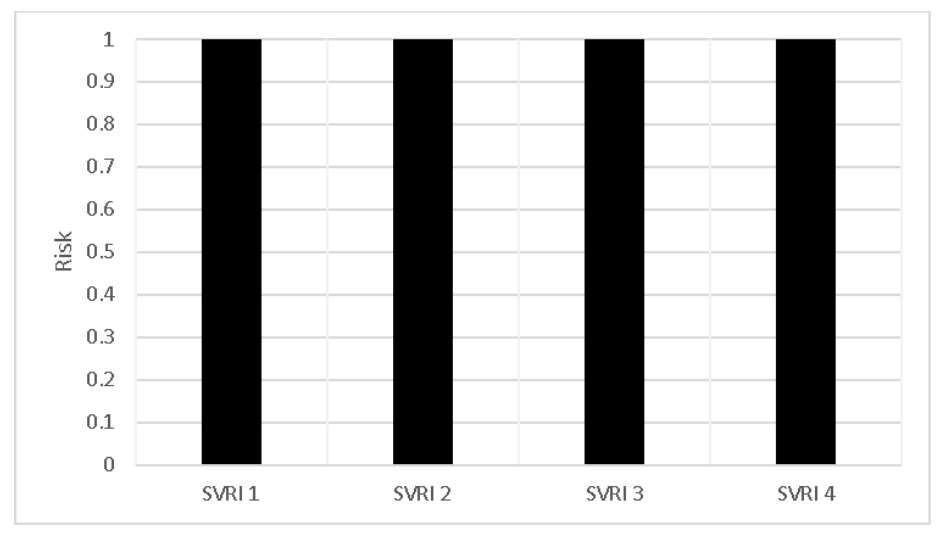

Figure 4. Visualizing SVRP censored notional raw data for an individual.

This modification was necessary to properly visualize the data in order to identify and prioritize risks for future clinical decision-making when data is not available as can been seen in the difference between Figures 3 and 4 where the censored data example produces significantly higher risks for all SVRI's than the original example.

With the development of Equation (10) and a process to visualize risks based on multiple exposures with multiple variables, two key questions still needed to be addressed:

1. Which variable $\left(v_{m, n}\right)$ was the most important? That is, should some variable(s) weigh more than others?

2. How do we account for the variability of each variable?

\subsection{Correction and Weighting Factors}

To answer the first question (e.g., is employment more important than medical history?), the equation was enhanced with a multiplicative "Weighting Factor", $\mathrm{WF}_{m, n}$, a numerical value that would account for importance of each variable $\mathrm{v}_{m, n}$. To account for the variability (confidence), the equation included a multiplicative "Correction Factor", $\mathrm{CF}_{\mathrm{m}, \mathrm{n}}$ a numerical value that would address the variability of each variable based on the confidence in the process, procedures, methods, device, etc. for each $\mathrm{v}_{m, n}$. Combining the CF with the WF resulted in the following iteration of the SVRP:

$$
\begin{gathered}
\text { SVRP }=\left[\begin{array}{c}
S V R I_{1} \\
\vdots \\
S V R I_{M}
\end{array}\right]= \\
{\left[\begin{array}{ccc}
\left(\mathrm{V}_{1,1}\right)\left(\mathrm{NF}_{1,1}\right)\left(\mathrm{CF}_{1,1}\right)\left(\mathrm{WF}_{1,1}\right) & \cdots & \left(\mathrm{v}_{1, \mathrm{~N}}\right)\left(\mathrm{NF}_{1,1}\right)\left(\mathrm{CF}_{1,1}\right)\left(\mathrm{WF}_{1, \mathrm{~N}}\right) \\
\vdots & \ddots & \vdots \\
\left(\mathrm{V}_{\mathrm{M}, 1}\right)\left(\mathrm{NF}_{1,1}\right)\left(\mathrm{CF}_{1,1}\right)\left(\mathrm{WF}_{\mathrm{M}, 1}\right) & \cdots & \left(\mathrm{v}_{\mathrm{M}, \mathrm{N}}\right)\left(\mathrm{NF}_{1,1}\right)\left(\mathrm{CF}_{1,1}\right)\left(\mathrm{WF}_{\mathrm{M}, \mathrm{N}}\right)
\end{array}\right] \cdot\left[\begin{array}{c}
1 \\
\vdots \\
1
\end{array}\right]}
\end{gathered}
$$


and simplifying the notation by observing the different factors from their own matrices. That is,

$$
\begin{gathered}
S V R P=\left[\begin{array}{c}
S V R I_{1} \\
\vdots \\
S V R I_{M}
\end{array}\right]= \\
\left(\left[\begin{array}{ccc}
v_{1,1} & \cdots & v_{1, N} \\
\vdots & \ddots & \vdots \\
v_{M, 1} & \cdots & v_{M, N}
\end{array}\right] \odot\left[\begin{array}{ccc}
N F_{1,1} & \cdots & N F_{1, N} \\
\vdots & \ddots & \vdots \\
N F_{M, 1} & \cdots & N F_{M, N}
\end{array}\right] \odot\right. \\
\left.\left[\begin{array}{ccc}
C F_{1,1} & \cdots & C F_{1, N} \\
\vdots & \ddots & \vdots \\
C F_{M, 1} & \cdots & C F_{M, N}
\end{array}\right] \odot\left[\begin{array}{ccc}
W F_{1,1} & \cdots & W F_{1, N} \\
\vdots & \ddots & \vdots \\
W F_{M, 1} & \cdots & W F_{M, N}
\end{array}\right]\right) \cdot\left[\begin{array}{c}
1 \\
\vdots \\
1
\end{array}\right]
\end{gathered}
$$

The SVRP can be written in a more compact form as

$$
S V R P=[\boldsymbol{V} \odot N F \odot C F \odot W F] \cdot[\mathbf{1}]
$$

Where $V$ is the matrix of the risk values, $N F$ is the matrix for the normalization factor values, $C F$ is the matrix for the correction factor values, $W F$ is the matrix for the weight factor values, and 1 is the column vector of all ones. Note that the SVRP in its simplest form, can be visualized as in prior examples/figures. However, to represent Equation (20) numerically required a process to convert the resulting column vector to a number. This is presented in Section 4.

\section{NUMERICAL REPRESENTATION OF THE SVRP}

To represent the SVRP numerically, one way is to average the SVRIs. Assume there are M negative outcomes then

$$
\operatorname{avg}(S V R P)=\frac{1}{M} \sum_{m=1}^{M} S V R I_{\mathrm{m}}
$$

Applying this to the previous example (see Equation (17) and Figure 3) then

$$
\operatorname{avg}(S V R P)=\frac{1}{4} \sum_{m=1}^{4} S V R I_{\mathrm{m}}=\frac{1}{4}(1+0.66+0.66+0.33)=0.6625
$$

is the overall risk for the individual with the ability to represent the SVRP numerically so the healthcare and/or social benefits providers can now use a numerical value for an individual transitioning Service member or Veteran's overall risk between 0 and 1, which could be represented as:

$$
\operatorname{avg}(S V R P)=\left\{\begin{array}{c}
0.00 \leq \mathrm{risk}<0.25 \text { is Low Risk } \\
0.25 \leq \mathrm{risk}<0.75 \text { is Med Risk } \\
0.75 \leq \mathrm{risk}<1.00 \text { is High Risk }
\end{array}\right\} .
$$

For example, the individual in Equation (22) would be rated at medium risk. Therefore, with a gauge of overall individual risk, the DoD/VA clinicians and/or social benefits advisors can easily triage multiple individuals allowing the organization or the healthcare/social benefits provider to prioritize care as warranted from high-to-low risk. Additionally, the healthcare and/or social benefits provider can use the SVRP visualization to identify an individual's highest negative outcome risk to prioritize and mitigate the risks. 
Of equal value to the DoD and VA is the capability to represent the SVRP with a single number which creates the ability to aggregate multiple SVRPs population analysis for health policy and resource decisions at a universal level.

\section{ApPlications}

With the SVRP fully constructed and simplified for an individual transitioning Service member/Veteran, healthcare and/or social benefits providers now have a tool that can be used to identify and prioritize individuals either numerically or visually. We can also expand the use of the SVRP for $n$ individuals with similar or different negative outcomes where one would expect to see $n$ distinct SVRPs. Therefore, observing a collection of SVRPs,

$$
\{\mathrm{SVRP} 1, \mathrm{SVRP} 2, \ldots, \mathrm{SVRP} n\}
$$

will also be of interest. For example, if we visualize the SVRPs for two individuals $n=2$ (see Figure 5), individual A has a high risk for suicide whereas individual B has a high risk for homelessness. This visualization allows the "healthcare and/or social benefits provider" to target and prioritize interventions based on the individual's highest potential risk of negative outcome(s).

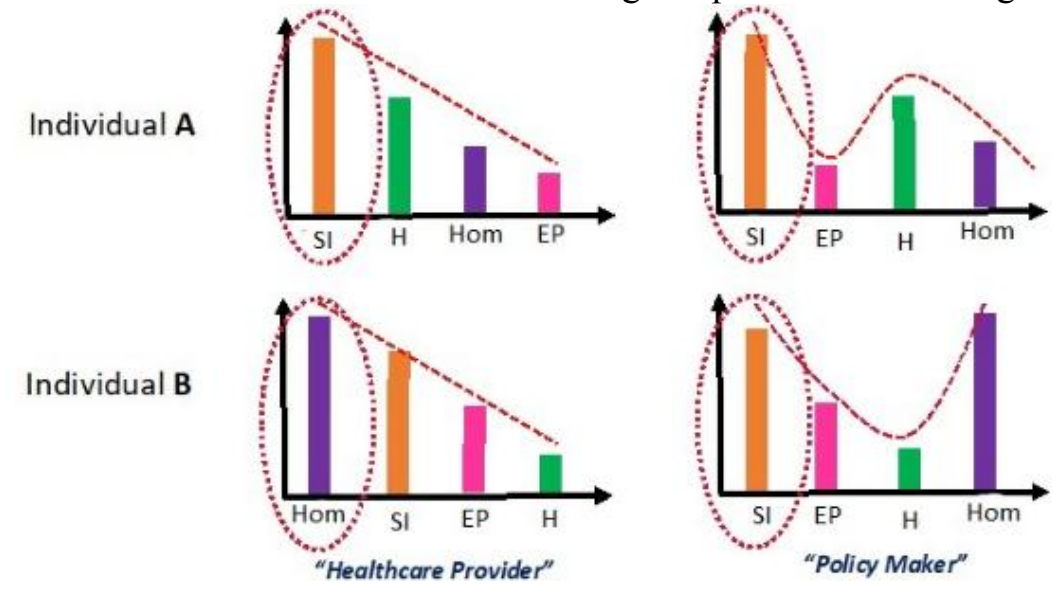

Figure 5. SVRP Benefits: Tailorability for healthcare providers and policy makers.

EP = Episodic Psychosis, H = Health, Hom = Homelessness, SI = Suicide Ideation.

But if we modify the visualization with the same data to accommodate a policy maker, we notice suicide is prevalent in both $\mathrm{A}$ and $\mathrm{B}$, allowing the policy maker to identity the high priority negative outcome for either policy development/modification or proper resourcing versus focusing on a lower priority exposure.

Note for consistency and broader use, it is crucial that every vm,n for an SVRI be the same when creating an SVRP. For example, if SVRI1 has been defined for a suicide exposure or SVRI1 = SVRIsuicide, then the $\mathrm{v} 1,1$ position in the matrix array will always be the poverty status measure for SVRIsuicide, the v1,2 position will always be the unemployment status for SVRIsuicide, and the $\mathrm{v} 1,3$ position will always be the clinical evidence in the individual's medical record for SVRIsuicide. The same would apply if SVRI2 is for a homelessness, SVRIhomelessness, SVRI3 is for 1st episode psychosis, SVRI lst episode psychosis, and so on for an SVRI1 to SVRIM. Appreciating the example in Figure 5 provides value for healthcare/social benefits providers at the individual level and policy makers at the aggregate level, now consider collating the overall risks (numerical solutions) for multiple SVRPs derived from Equation (21) for very large populations. 
This can be visualized using a notional data for a subpopulation for Veterans, for example, the VA's healthcare regions (North East, South East, North West, South West). Populating the $\operatorname{avg}($ SVRP) for every individual in the hypothetical VA's regional population with notional data, we can visualize the negative outcomes of the entire VA population in Figure 6.

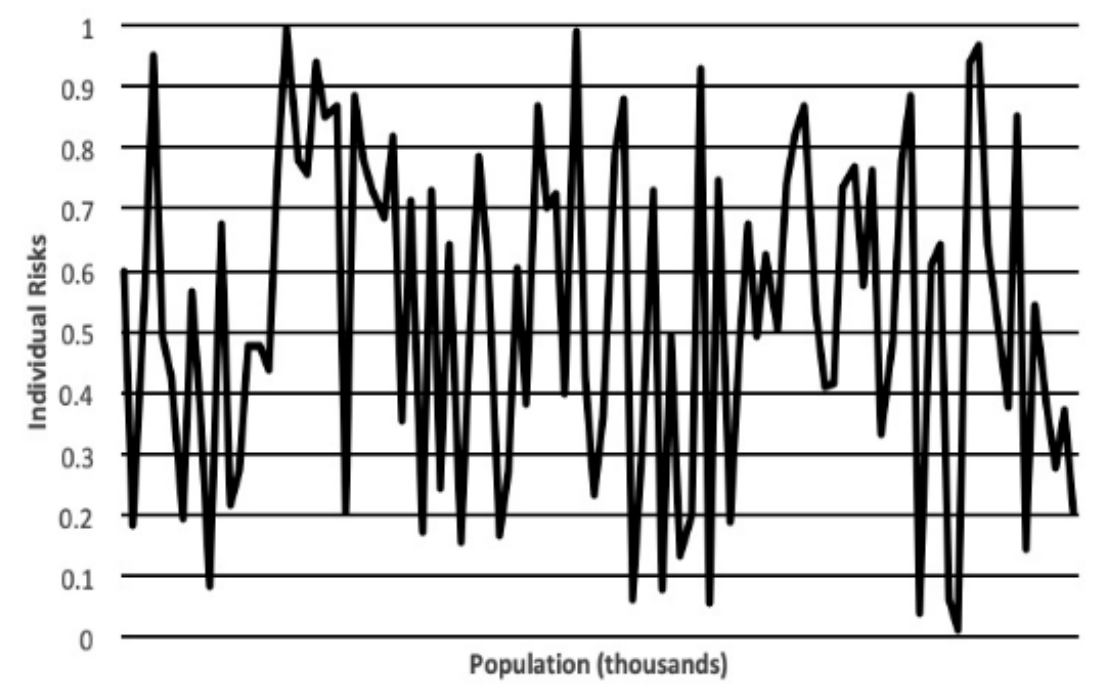

Figure 6. Notional representation of aggregated avg(SVRP)s for a large population (e.g., US Veteran population).

Visually, this can be overwhelming, so if we rearrange the risk values in descending order we get a more practical visualization for SVRP population curve (see Figure 7).

However, to understand the overall risk of the population and/or to make comparisons between populations it makes sense to create a qualitative measure for each population curve. This can be represented by the area under the curve (AUC) of the graph in Figure 7. It turns out that the AUC equals the average across the population.

Suppose we wish to compare two different populations A and B. If the $\mathrm{AUC}(\mathrm{A})<\mathrm{AUC}(\mathrm{B})$ then population B has a higher overall risk. The AUC is computed using (21) for each individual SVPRa in population $\mathrm{A}$ and $\mathrm{SVPRb}$ in population $\mathrm{B}$. That is,

$$
\operatorname{AUC}(\mathrm{A})=\frac{1}{|\mathrm{~A}|} \sum_{a \in A} \operatorname{SVRPa}=\operatorname{avg}(\operatorname{SVRP}(A))
$$

Where $|\mathrm{A}|$ denotes the cardinality (the size or number) of the population $\mathrm{A}$, and a is an individual from the population A. Similarly, for population B,

$$
A U C(\mathrm{~B})=\frac{1}{|\mathrm{~B}|} \sum_{b \in B} \operatorname{SVRPb}=\operatorname{avg}(\operatorname{SVRP}(B)) .
$$

We can generalizing to any population

$$
\operatorname{AUC}(\mathrm{X})=\frac{1}{|\mathrm{X}|} \sum_{x \in X} \operatorname{SVRPx}=\operatorname{avg}(\operatorname{SVRP}(X))
$$


Health Informatics - An International Journal (HIIJ) Vol.10, No.2, May 2021

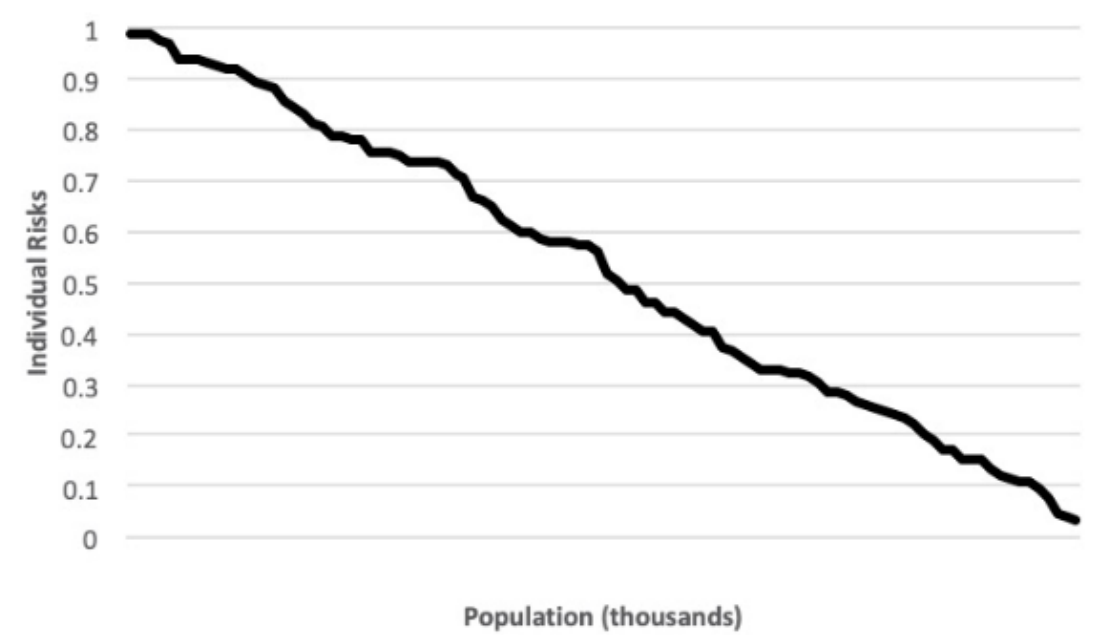

Figure 7. Rearranging aggregated avg(SVRP)s for a large population (e.g., US Veteran population).

Using Equation (27) the AUC(X) in Figure 7 is 0.526 . This numerical representation for the overall negative out-come risks of the US veteran population can easily be compared to other regions or partitioned for deeper analysis into various VA regions for comparison between the regions. More importantly, decision makers can further examine the raw data to:

1) Identify which negative outcomes are most prevalent,

2) Identify the individuals or groups at elevated risk, or

3) Identify groups of individuals that require more evaluation and proper funding for high risk areas to mention a few uses.

\section{DISCUSSION}

Key for the success of the SVRP will be the monitoring, collection, identification, and understanding of different types of negative outcome and the integration of multiple data streams into a unified framework that can provide useful information to improve transitioning Service member/Veteran outcomes. Additionally, several ethical and privacy-related issues must be navigated as the SVRP is implemented. The most complex of these issues involve identifiably, the increasingly sensitive nature of mental health information, and the limits of confidentiality.

But before the SVRP can be implemented, further studies will be needed to validate and refine the mathematical expression/process to develop statistically validated algorithms to determine health risk profiles and drive preventative strategies for individuals. Once validated, an implementation plan to apply individual data to SVRP models that result in risk profiles to inform individuals for preventative behaviors and improved out- comes will be established. This includes ensuring all data is collected using validated, approved methods and analyzed with appropriate quality and regulatory requirement for use in the individual's health record. In the long-term, the SVRP will translate these findings into clinically and non-clinically actionable recommendations improving TSM/Veteran well-being.

\section{Conclusions}

Bold ideas and constructs require sound foundations. In the case of the SVRP it became imperative to develop the mathematical expression and process to describe the construct: the first to combine 
clinical and social determinants into one individual risk profile. With the math the SVRP can now be turned into machine language and developed into a modern tool to better understand the effects of transiting service member from the military into civilian life decreasing the negative outcomes associated with transition and improving TSM/Veteran's overall quality of life.

Though early in its development, the SVRP displays individual and population risks through userfriendly visualizations and quantitative values. Ultimately this framework will be implemented as part of a future Decision Support System (CDSS) within the DoD/VA healthcare system's Electronic Health Record, where the SVRP seamlessly ingest clinical and social determinant data at the individual level and calculates personal risk profiles where the aggregate information can be used to inform and guide decision makers to make more informed policy and resource decisions.

\section{REFERENCES}

[1] Derefinko, Karen J., Troy A. Hallsell, Matthew B. Isaacs, Francisco I. Salgado Garcia, Lauren W. Colvin, Zoran Bursac, Meghan E. McDevitt-Murphy, James G. Murphy, Melissa A. Little, Gerald W. Talcott, Robert C. Klesges, Substance Use and Psychological Distress Before and After the Military to Civilian Transition, Military Medicine, Volume 183, Issue 5-6, May-June 2018, Pages e258-e265, https://doi.org/10.1093/milmed/usx082

[2] Mansfield, A. J., Bender, R. H., Hourani, L. L. and Larson, G. E. (2011), Suicidal or Self-Harming Ideation in Military Personnel Transitioning to Civilian Life. Suicide and Life-Threatening Behavior, 41: 392-405. https://doi.org/10.1111/j.1943- 278X.2011.00039.x

[3] Ross, Christina L. 2009. Integral Healthcare: The Benefits and Challenges of Integrating Complementary and Alternative Medicine with a Conventional Healthcare Practice. Integrative Medicine Insights 4:13-20. https://doi.org/10.4137/imi.s2239.

[4] The Institute of Medicine (IOM). Disparities in Health Care: Methods for Studying the Effects of Race, Ethnicity, and SES on Access, Use, and Quality of Health Care, 2002.

[5] Ravindran C, Morley SW, Stephens BM, Stanley IH, Reger MA. Association of Suicide Risk With Transition to Civilian Life Among US Military Service Members. JAMA Netw Open. 2020;3(9):e2016261. doi:10.1001/jamanetworkopen.2020.16261

[6] Vogt D., Taverna, E., Nillni, Y., Tyrell, F. (2018). Manual for the Well-Being Inventory (WBI): A multidimensional tool for assessing key contributors to well-being. Boston, MA: National Center for PTSD.

[7] Horn, Roger A., Charles R. Johnson, (1994). Topics in Matrix Analysis. Cambridge: Cambridge University Press

[8] DHHS 2017, Poverty Guidelines for the 48 Contiguous States and the District of Columbia, Office of the Assistant Secretary for Planning and Evaluation, https://aspe.hhs.gov/2017-povertyguidelines\#threshholds.

[9] Stack, S. 2000. Suicide: A 15-year review of the sociological literature part I: Cultural and economic factors. Suicide \& Life - Threatening Behavior 30, no. 2:145-62.

[10] Reams, Robert. 1999. Hadamard Inverses, Square Roots and Products of Almost Semidefinite Matrices." Linear Algebra and Its Applications 288:35-43. https://doi.org/.10.1016/S00243795(98)10162-3

\section{AUTHORS}

Mark E. Oxley, PhD: Professor of Mathematics, Department of Mathematics and Statistics, Graduate School of Engineering and Management, Air Force Institute of Technology, Wright-Patterson, Air Force Base, Ohio, USA

Richard T. Hartman, PhD: Executive Director, Office of Transition and Economic Development, Veterans Benefits Administration, Department of Veterans Affairs, Washington, DC, USA 